\title{
DIMENSIONS OF KNOWLEDGE IN PROTOTYPING: A REVIEW AND CHARACTERISATION OF PROTOTYPING METHODS AND THEIR CONTRIBUTIONS TO DESIGN KNOWLEDGE
}

\author{
Real, Ricardo; \\ Snider, Chris; \\ Goudswaard, Mark; \\ Hicks, Ben \\ University of Bristol
}

\begin{abstract}
Whilst prior works have characterised the affordances of prototyping methods in terms of generating knowledge about a product or process, the types, or 'dimensions' of knowledge towards which they contribute are not fully understood. In this paper we adapt the concept of 'design domains' as a method to interpret, and better understand the contributions of different prototyping methods to design knowledge in new product development. We first synthesise a set of ten dimensions for design knowledge from a review of literature in design-related fields. A study was then conducted in which participants from engineering backgrounds completed a Likert-type questionnaire to quantify the perceived contributions to design knowledge of 90 common prototyping methods against each dimension. We statistically analyse results to identify patterns in the knowledge contribution of different methods. Results reveal that methods exhibit significantly different contribution profiles, suggesting different methods to be suited to different knowledge. Thus, this paper indicates potential for new methods, methodology and processes to leverage such characterisations for better selection and sequencing of methods in the prototyping process.
\end{abstract}

Keywords: Design cognition, Design practice, New product development, Design learning

\section{Contact:}

Real, Ricardo

University of Bristol

Faculty of Engineering

United Kingdom

ric.real@bristol.ac.uk

Cite this article: Real, R., Snider, C., Goudswaard, M., Hicks, B. (2021) 'Dimensions of Knowledge in Prototyping: A Review and Characterisation of Prototyping Methods and Their Contributions to Design Knowledge', in Proceedings of the International Conference on Engineering Design (ICED21), Gothenburg, Sweden, 16-20 August 2021. DOI:10.1017/ pds. 2021.130 


\section{INTRODUCTION}

The creation of a prototype is a primary mediator between the designers cognitive model of a concept and its materialisation in the physical world, Camere and Bordegoni (2016) describe this as an engagement with the 'product-to-be', querying the many elements inherent to an artefacts realisation. As such, prototyping is regarded a critical activity in New Product Development (NPD) (Wall, Karl T. Ulrich, and Flowers, 1992) with a multitude of methods, and methodology encompassed within the creation of prototypes; each influencing the nature of information and learning generated in the process (Gero, 1990). Prototypes, ranging from low to high fidelity, are frequently iterated throughout the NPD process (Buchenau and Suri, 2000) adopting various roles to probe a particular design challenge, or opportunity (Camburn et al., 2017). Ullman (1992) classifies such prototypes into proofs of Concept, Product, Process, and Production.

However, Bogers and Horst (2014) identify an understanding of prototyping to exist simultaneously at different levels, an organisational level, and informal designer level where the tangible practices of prototyping take place. Whilst the affordances of prototyping are generally understood organisationally, the informal fuzzy front end of design lacks as clear a definition with designers often finding it difficult to know which methods to use, and when to employ them in the design process (Lim, Stolterman, and Tenenberg, 2008). This suggests a differentiation between the types of knowledge implicit in prototyping, the designer requiring an imperative, procedural knowledge of processes and methods, as opposed to a descriptive knowledge for more formal project management and operations (Engstrom, 2009). Prior works allude to a particular set of dimensions in which knowledge is required to realise a concept. Karl T Ulrich (2003) defines the prototype as an approximation of the product along one or more dimensions of interest, whilst Schon and Wiggins (1992) suggest a causality amongst dimensions, with actions in one dimension having consequences in all others. It's therefore hypothesised that by identifying these dimensions in prototyping, and further characterising the contributions to design knowledge afforded by various prototyping methods that designers could better select appropriate methods, and query specific dimensions of interest, thus, supporting design processes and accelerating product development. To this end, this paper aims to investigate and characterise differences in the knowledge contribution of different prototyping methods, following which it will discuss the opportunities for application and how this information may be used to better structure the prototyping process.

This paper begins by defining a set of knowledge dimensions for design prototyping, extracted from related works. Following, it presents a study in which physical and digital prototyping methods at varying fidelity were rated for their contribution to each knowledge dimension. Results were analysed statistically using Friedman's ANOVA and post-hoc Dunn-Bonferroni pairwise comparisons to detect differences in knowledge contributions of methods, and emergent patterns between method types. Finally, the paper reflects on the impact of varying knowledge on prototype selection and sequencing, and identifies opportunities for future work.

\section{RELATED WORK}

Prior works, particularly that of Schon and Wiggins (ibid.) in the study of architecture describe the process of design as a reflective conversation with the materials of a design solution. The example of a designer working in the medium of drawing is given, "the designer sees what is 'there' in some representation of a site, draws in relation to it, and sees what has been drawn, thereby informing further designing" the designer not only registers visual information, but identifies and gives meaning to patterns beyond the medium itself.

Schon further outlines a set of 'design domains' in which these acts of making and 'seeing' ${ }^{1}$ register to the different necessary elements in architectural design. These include Programme use, Siting, Building elements, Organisation of space, Form, Structure/Technology, Scale, Cost, Building Character, Precedent, Representation, and Explanation. To develop the design, the designer must develop their understanding in each of these domains and, as learning is tied to the activity, there is suggestion that the media and activity of its use may substantially impact design development.

Prototypes in NPD take many forms with broad use, ranging from highly conceptual to near-final proofof-production models (Buchenau and Suri, 2000). Such prototypes may range from analytic (virtual)

${ }^{1}$ Wittgenstein (2009) 'Seeing as' judgement of a pattern as 'seen' in the first sense. 
to physical models, and may test individual components through to comprehensive systems (Karl T Ulrich, 2003). In so doing, prototypes will develop and test different aspects of the design (i.e. the role, implementation, and look/feel prototypes of Houde and Hill (1997)) for a range of process goals (Camburn et al., 2017), at a level appropriate to the process stage and required fidelity (Lim, Stolterman, and Tenenberg, 2008).

Given such breadth of method and purpose, that a set of design domains must be informed against to produce a design, that learning is to some degree influenced by media, and that designers often do not know which prototyping techniques to choose (ibid.), a question arises as to the inter-relation between method, type, and knowledge produced. This paper aims to investigate this relationship through characterisation of the 'design domains' to which different prototyping methods contribute, and exploration of how knowledge of such contributions may support better structuring of the prototyping process.

\section{METHOD}

To characterise this relationship, a study was performed in which participants were asked to rate a variety of prototyping methods for their contribution towards the 'types' of knowledge required to fully develop a product. This section details the method followed in the paper, the study materials provided to each participant and outlines the process participants followed in the study.

\subsection{Extraction of Knowledge Dimensions from literature}

As the 'design domains' of Schon and Wiggins (1992) are situated within the architecture domain, it was first necessary to recontextualise to NPD. Table 1 provides definitions of each as mapped to the NPD domain. To provide differentiation against Schon and recognising differences in terminology of the fields, these design domains are hereon termed Knowledge Dimensions.

Table 1. Knowledge dimensions, adapted fromSchon and Wiggins (1992)

\begin{tabular}{lll}
\hline ID & Knowledge Dimension & Description \\
\hline KD1 & Programme Use & What the design is intended to do (i.e. its function) \\
KD2 & Environment & How the design performs within its intended conditions of use \\
KD3 & Resources & What is needed to make the design (e.g. materials, components, tools, time, cost) \\
KD4 & Design Elements & Identification of the features or components that will comprise the design \\
KD5 & Form & The shape and size of the design including how it looks and feels \\
KD6 & Manufacturing Processes & How the design will be made, the steps and tools required to make it \\
KD7 & Configuration & The arrangement of features and components, how the design fits together \\
KD8 & Character & How the design is supposed to look (including any context of brand and/or product family) \\
KD9 & Explanation & How the prototype, or elements of, communicate what it does \\
KD10 & Lifecycle & The envisaged life of the design, including its creation, use and disposal \\
\hline
\end{tabular}

\subsection{Study Materials}

Each participant was provided with a rating spreadsheet comprised of a structured table of prototyping methods against which they were asked to rate each knowledge dimension. Methods were to be rated on a 5-point Likert scale by the degree to which participants thought the method would inform each respective knowledge dimension. Participants were additionally asked to consider prototyping in the context of a single product and briefed using an example cordless drill; a labelled drawing of the drill was included with study materials and available as reference throughout. Generation of study materials required extraction of knowledge dimensions from literature, and generation of a list of prototyping methods which will be detailed in the following sections. A snapshot of the rating spreadsheet is shown in Figure 1.

\subsection{Formulation and categorisation of prototyping methods}

Prototyping incorporates a wide array of methods with varying properties and purposes (Karl T Ulrich, 2003). While it is unfeasible to generate an exhaustive list of potential methods, it is important that those categorised within the study represent a breadth of the characteristics of interest within the study. 


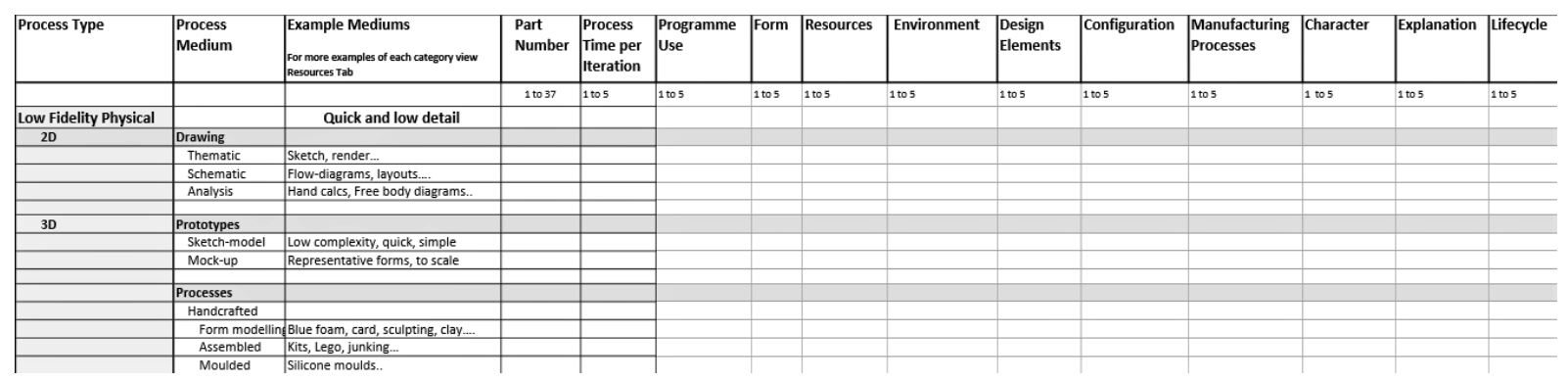

Figure 1. Extract from rating sheet

A list of 90 common prototyping methods was generated by the researchers based on literature and experience. This list was reviewed by study participants prior to the study itself, who were asked to suggest additional methods according to their own knowledge. The summary categories of extracted methods are shown in Table 3. Following generation, methods were classified by the same researchers according to the categories presented in Table 2. These categories were selected to support analysis and align with wider research aims, see Section 1, namely spanning the prominent digital / physical boundary in prototyping methods (Karl T Ulrich, 2003), recognising that fidelity of prototyping may vary depending on process stage and activity, and that both method domain (physical / digital) and level of fidelity required may impact method choice in NPD. Although they were categorised, participants rated each method individually. For brevity, Table 3 presents only categories and examples.

Table 2. Prototyping method classifications

\begin{tabular}{ll}
\hline Category & Description \\
\hline $\begin{array}{l}\text { Physical or Digital } \\
\text { Level of Fidelity }\end{array}$ & $\begin{array}{l}\text { Whether the method primarily comprises physical or digital matter } \\
\text { (Low or High) The degree to which dimensional precision is controlled for the method }\end{array}$ \\
\hline
\end{tabular}

Table 3. Prototyping method categories. All methods may be completed at a low or high fidelity.

\begin{tabular}{lll}
\hline Name & Physical/Virtual & Description \\
\hline Hand Processes & Physical & Any manual process implemented by the designer (e.g. foam modelling, card modelling) \\
Machine Fabrication & Physical & Any machine-based process manually controlled by the designer (e.g. turning, milling, cutting, joining) \\
CNC Machining & Physical & Any CNC controlled machining process including additive, subtractive and pattern cutting processes \\
Finishing & Physical & Any surface finishing process carried out by the designer (manual, or with machine tool assistance) \\
2D Drawing & Physical & Any drawing process, including thematic (e.g. sketch, rendering), schematic (e.g. technical drawing, layout), \\
& & and analytic (e.g. free body diagram) \\
2D Drawing & Virtual & Any computer based 2D drawing process (including categories as listed above) \\
3D Model Generation & Virtual & Generation of any 3D geometry \\
Visualisation & Virtual & Generation of renders, visualisations, and representations of as-final geometry \\
\hline
\end{tabular}

\subsection{Study Process}

Six participants partook in the study. Participants were all design engineers and active researchers in the department of Mechanical Engineering, with expertise in prototyping processes. Participant design/engineering industry experience was captured prior to commencing the study, with three participants indicating 5+ years experience, two participants between 1 and 5 years, and one participant noting 'other' industry experience. The study comprised of two parts, first a participant briefing to familiarise participants with study materials, and the prototyping method rating.

Participant Briefing Participants were provided with a study briefing document defining all elements included within the rating spreadsheet, and providing examples of knowledge dimensions and prototyping methods. An initial meeting was held prior to rating with all participants, where the purpose of the study was explained, all rated elements were defined, and participants were able to request clarification. This process supported development of shared rater understanding. 
Prototyping Method Rating Participants were requested to rate each prototyping method against each knowledge dimensions on a 5-point Likert scale (1 - little or no information, 2, 3, 4, 5 - lots of information). Of note is that participants rated each individual method against each knowledge dimensions rather than the method category as a whole, where for brevity Table 3 shows categories only (i.e. thematic, schematic, and analytic drawings were all separately rated). Participants were not given a time limit for their rating. All participants completed all sections of the rating sheet. Once complete, participants returned their sheets to the researcher for collation, processing, and analysis.

\section{RESULTS}

Once collected, survey results from each participant were collated into a single working sheet, and median values for each category were extracted. Results were analysed to explore differences between prototyping methods with respect to their contribution to knowledge dimensions, first through quantitative detection of difference, and second through characterisation of these differences. The results comprise of two sections, the first compares knowledge dimensions across prototyping methods (Section 4.1) and the second identifies and explores differences between prototyping methods (Section 4.2). All statistical tests were carried out with IBM SPSS 27. Note that throughout this section, knowledge dimensions are referred both by name and ID as stated in Table 1 .

\subsection{Knowledge Dimensions across prototyping methods}

Rated values for each knowledge dimension were analysed using SPSS through a non-parametric Friedman's repeated measures test, showing a statistically significant difference $\left(\chi^{2}(9)=288.390, p<\right.$ $0.001)$ in the distribution of knowledge gained from different prototyping methods against the different dimensions.

Following the Friedman test a post hoc analysis was carried out via means of a pairwise DunnBonferroni comparison to detect further quantitative difference in knowledge contributions to each of the dimensions. The results for this are shown in Table 4. The results again show a statistical difference in the contribution of methods to different knowledge dimensions, with a typcially higher contribution to form (KD5) and programme use (KD2), and a lower contribution to lifecycle (KD10), explanation (KD9), and manufacturing processes (KD6). Where knowledge against all dimensions is necessary for completion of a design, this suggests that not all methods are equally appropriate.

Table 4. Knowledge dimension ranking.

\begin{tabular}{lll}
\hline & Knowledge Dimension & Mean Rank \\
\hline 1 & Form (KD5) & 6.49 \\
2 & Programme Use (KD1) & 6.18 \\
3 & Configuration (KD7) & 5.91 \\
4 & Design Elements (KD4) & 5.87 \\
5 & Character (KD8) & 5.34 \\
6 & Resources (KD3) & 5.29 \\
7 & Environment (KD2) & 5.26 \\
8 & Manufacturing Processes (KD6) & 5.11 \\
9 & Explanation (KD9) & 5.06 \\
10 & Lifecycle (KD10) & 4.49 \\
\hline
\end{tabular}

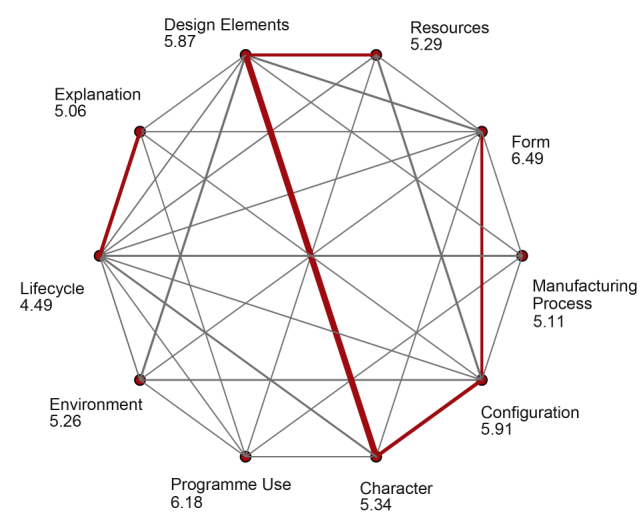

Figure 2. Pairwise comparison of knowledge dimensions

Additionally, results from the pairwise analysis indicate a measure of non-separability between dimensions, as observed in the graph of pairwise comparisons (fig.2). The results suggest interdependence between dimensions, showing a greater interdependence between dimensions illustrated in red, such as Design elements (KD4)/Character (KD8), and Explanation (KD9)/Lifecycle (KD10) highlighted by the distribution of prototyping method contributions in the analysis.

Table 5 lists the three prototyping methods that were rated as contributing to the highest degree to each knowledge dimension. Difference between knowledge dimensions is evident, with (e.g.) programme 
Table 5. Top-3 methods for each knowledge dimension. PIV indicate physical or virtual, LF/HF indicate low or high fidelity (no index indicates both)

\begin{tabular}{llll}
\hline Dimension & Top 3 Prototyping Methods & \\
\hline Programme Use & P: CNC Additive(AM) HF & P: Fabrication LF & Analysis Drawings HF \\
Form & P: CNC Subtractive(S) HF & P: Moulding HF & P: CNC AM HF \\
Resources & V: CAM HF & P: Moulding HF & Schematic Drawing HF \\
Environment & V: 3D Visualisation HF & P: Fabrication HF & P: CNC Subtractive HF \\
Design Elements & P: Drawing HF & V: CAD HF & P: Fabrication LF \\
Configuration & Schematic Drawing HF & V: CAD HF & V: 3D Visualisation HF \\
Manufacturing Process & V: CAM & P: CNC AM/S HF & P: Moulding HF \\
Character & V: 3D Visualisation HF & P: Drawing HF & P: Finishing HF \\
Explanation & Drawing HF & V: Visualisation & P: Form Modelling LF \\
Lifecycle & V: CAM HF & P: Fabrication & P: Moulding HF \\
\hline
\end{tabular}

use (KD1) and form (KD5) best informed by physical methods at high fidelity, while all other knowledge dimensions show a mixture of physical and virtual. The following section investigates per-method difference in detail.

\subsection{Differences between prototyping methods}

To identify high level differences between prototyping methods, each was grouped according to the categories given in Table 2 (Physical or Digital, and Level of Fidelity). This was done to test the hypothesis that each may have a significant impact on the efficacy of prototyping media against the knowledge dimensions to which they contribute. Figure 3 shows the contribution of grouped methods to knowledge dimensions.

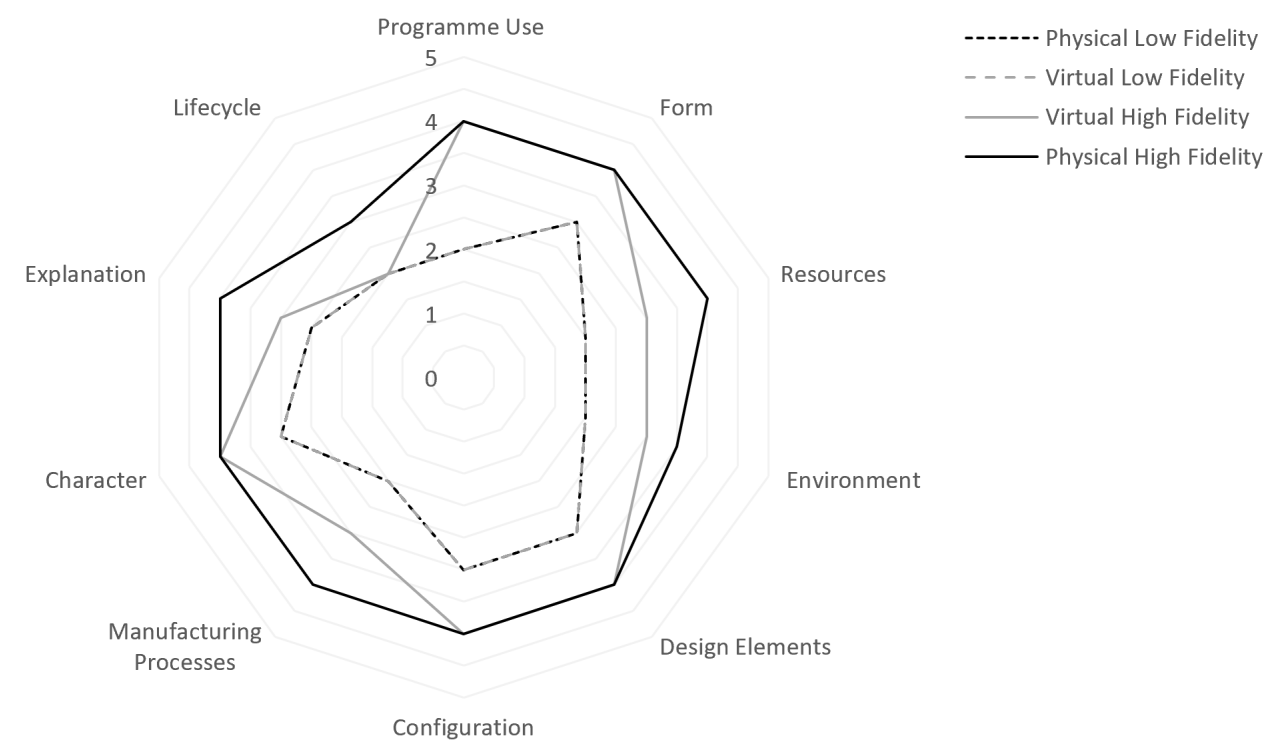

Figure 3. Prototyping method categories and their contribution to knowledge dimensions (5 showing a high contribution)

Several results are evident. First, low fidelity processes, whether physical or digital, contribute to a lower degree against all knowledge dimensions when compared to their high fidelity counterparts. However an exception to this is observed in lifecycle (KD10), where both high and low fidelity virtual processes perform poorly. Secondly, physical processes consistently score higher to virtual across fidelities, with the exceptions of programme use (KD1), form (KD5), design elements (KD4), configuration (KD7), and character (KD8) where physical and virtual scores align. It is of note that those where alignment 
occurs include dimensions more concerned with the geometry and layout of the prototype, than its wider resource use, environment, and fabrication, where physical processes are considered more informative. Of interest is that virtual processes are not considered as informative in explanation (KD9), which concerns communication of the prototype and its use. As they are built towards specific purposes, this finding may align with a) their lack of breadth in communication, where the specificity of the virtual prototype restricts communication of its use as a whole product, and $b$ ) that building a virtual prototype to fully mimic prototype use is a substantial task and less commonly performed.

\section{Method-specific comparison}

Tables 6 and 7 categories of prototyping methods against knowledge dimensions, showing low fidelity methods and high fidelity methods respectively. Ratings shown are medians of participant answers with variance calculated through median absolute deviation. Due to the lower number of participants in this study, this variance lacks granularity and can be used for indication only.

Table 6. Median low fidelity prototyping method ratings against knowledge dimensions

\begin{tabular}{|c|c|c|c|c|c|c|c|c|c|c|}
\hline \multirow[t]{2}{*}{ Method Category } & \multicolumn{10}{|c|}{ Knowledge Dimensions } \\
\hline & Prog. Use & Form & Res. & Env. & Des.Elem. & Conf. & Man. & Char. & Exp. & Lifecycle \\
\hline P: Hand Processes & 3 & 3 & 2 & 2 & 2 & 2.5 & 2 & 3 & 2.5 & 1.5 \\
\hline P: Machine Fabrication & 2.5 & 3 & 3 & 2 & 2 & 2 & 3 & 2.5 & 2 & 2 \\
\hline P: Machine CNC & 2 & 3 & 2 & 2 & 2 & 2 & 2 & 2 & 2 & 1.5 \\
\hline P: Finishing & 2 & 2.5 & 2 & 2 & 2 & 1 & 2 & 3.5 & 2 & 1 \\
\hline P: 2D Drawing & 2.5 & 2 & 2 & 2 & 3 & 2.5 & 2 & 2 & 2 & 2 \\
\hline V: 2D Drawing & 2.5 & 2 & 2 & 2 & 3 & 2.5 & 2 & 2 & 2 & 2 \\
\hline V: 3D Model Generation & 2 & 3 & 2.5 & 2 & 2.5 & 3 & 2 & 3 & 2 & 2 \\
\hline Median & 2.5 & 3 & 2 & 2 & 2 & 2.5 & 2 & 2.5 & 2 & 2 \\
\hline Variance & 0.5 & 0 & 0 & 0 & 0 & 0.5 & 0 & 0.5 & 0 & 0 \\
\hline
\end{tabular}

Table 7. Median high fidelity prototyping method ratings against knowledge dimensions

\begin{tabular}{|c|c|c|c|c|c|c|c|c|c|c|}
\hline \multirow[t]{2}{*}{ Method Category } & \multicolumn{10}{|c|}{ Knowledge Dimensions } \\
\hline & Prog. Use & Form & Res. & Env. & Des.Elem. & Conf. & Man. & Char. & Exp. & Lifecycle \\
\hline P: Hand Processes & 2 & 4 & 2 & 2 & 2.5 & 2 & 2 & 3.5 & 3 & 1 \\
\hline P: Machine Fabrication & 3 & 4 & 3 & 2 & 2.5 & 3 & 3 & 3 & 2 & 3 \\
\hline P: Machine CNC & 2.5 & 3.5 & 3 & 2 & 2.5 & 2 & 3 & 3 & 2 & 2 \\
\hline P: Finishing & 2.5 & 3.5 & 3 & 2.5 & 2.5 & 2 & 3 & 3.5 & 3 & 2.5 \\
\hline P: 2D Drawing & 3 & 3 & 3 & 2 & 3.5 & 3 & 2 & 2 & 2.5 & 2 \\
\hline V: 2D Drawing & 3 & 2 & 2 & 2 & 3 & 3 & 2 & 2 & 3 & 2 \\
\hline V: 3D Model Generation & 3 & 4 & 3 & 2 & 3 & 3 & 3 & 3 & 2 & 3 \\
\hline V: Visualisation & 3 & 4 & 2 & 3 & 3 & 3.5 & 2 & 5 & 3.5 & 1 \\
\hline Median & 3 & 3.75 & 3 & 2 & 2.75 & 3 & 2.5 & 3 & 2.75 & 2 \\
\hline Variance & 0 & 0.25 & 0 & 0 & 0.25 & 0.25 & 0.5 & 0.5 & 0.5 & 0.75 \\
\hline
\end{tabular}

Findings align with data shown in Fig. 3 above, with high fidelity methods rated as more contributory to each knowledge dimension. Exceptions exist only for lifecycle (KD10) and environment (KD2) where both high and low fidelity were rated equally with a low score of 2 . Most knowledge dimensions show low variance across methods, particularly those of low fidelity, with only programme use (KD1), configuration (KD7), and character (KD8) showing a non-zero median variance. This suggests either that, in high level terms, many of the low fidelity prototyping methods are similarly capable across knowledge dimensions, or that the Likert scale and number of participants within the study did not provide sufficient data to discern a significant difference. This does not hold for high fidelity methods, where a majority of knowledge dimensions show variance, with only programme use (KD1), resources (KD3), and environment (KD2) clustered closely to central values. That some knowledge dimensions show higher variation, particularly manufacture (KD6), character (KD8), explanation (KD9), and lifecycle (KD10) for high fidelity (Table 7), and form (KD5), character (KD8), configuration (KD7) for low fidelity (Table 6) suggests a need for some care during method selection. There may prove a higher 
tendency for some methods to bias towards certain dimensions, and it may be likely that a requirement for multiple prototypes exist when generating high fidelity prototypes that contribute to all.

Looking more specifically at the scores of grouped methods, several findings may be suggested.

For low fidelity methods, (Table 6):

- Little appreciable difference is evident between methods with many showing very similar scores, as evidenced by a low variance. This could suggest the viability of many methods to contribute across knowledge dimensions during the low fidelity prototyping process.

- Both virtual and physical drawing methods are better aligned with design elements (KD4) and configuration (KD7), but rated less well against other dimensions. Both of these dimensions concern more informative aspects with less reliance on geometry, function, and motion, and perhaps are better suited to the informative representation style that drawings create. Should knowledge against other dimensions be desirable, drawings may prove a less advisable choice.

- Virtual model generation (i.e. CAD) is rated as capable or better than physical modelling methods in the majority of categories. This suggests that low fidelity virtual modelling methods, perhaps due to their flexibility in generation, viewing, and exploration, are well suited to prototype learning. The exception here is in programme use (KD1), perhaps highlighting that functional assessment of a low-fidelity prototype is difficult in the virtual domain.

- Hand-based processes (i.e. manually constructed by the designer) in general score more highly than machine-based process (either designer or computer controlled) in programme use (KD1), configuration (KD7), and character (KD8). This may be due to the tangible exploratory nature of hand fabrication and related cognitive processes, which require closer engagement with the feel of the prototype, its arrangement, and in-process evaluation of use during fabrication.

- Machine-based process are rated more highly in manufacturing (KD6) and resource (KD3), perhaps due to the natural closeness of machine manufacturing to the final manufacturing processes to be used during production.

Looking across high fidelity methods (Table 7), some similarities may be observed:

- As with low fidelity, high fidelity virtual model generation methods show fairly similar values to physical methods. It is apparent that the drop in virtual method capability when compared to physical models shown in Figure 3 are due to lower scores for virtual drawing and visualisation in specific dimensions, rather than general poor performance across all. For example, in manufacturing (KD6), resources (KD3), and lifecycle (KD10).

- $\quad$ The rating of hand-based process is relatively lower against machine-based processes, with lower scores in programme use (KD1), resources (KD3), configuration (KD7), and manufacture (KD6). This may be due to the importance placed on the physical form for utility in high fidelity prototyping, wherein machine fabrication methods are more likely to be of sufficient quality to be useful (programme use (KD1)), and are representative of as-final manufacture (resources (KD3) and manufacture (KD6)). Physical finishing and virtual visualisation - both methods of ensuring as-final representation of the design, rate slightly higher against character (KD8), explanation (KD9), and environment (KD2) than other methods. As methods that are closer aligned with communicating as-final representation and use this is logical.

- As with low fidelity, the least generally useful methods include drawing in both physical and virtual. While some parity to others remains in design elements (KD4) and configuration (KD7), drawings are considered substantially worse against form (KD5), perhaps due to their 2D nature and need for cognitive interpretation.

- Machine fabrication methods and virtual 3D modelling methods are rated as apparently strongest across the knowledge dimensions, perhaps due to their precision against the design and viability for use in evaluation, communication, and learning. For cross-dimension completeness, these methods may prove a preferred choice.

\section{Discussion}

These findings, while exploratory, present several potential implications for design prototyping and selection of methods. 
Contribution of methods to knowledge dimensions: It is evident from the findings that different prototyping methods contribute to each knowledge dimension to differing degrees. Given the range of prototyping methods that may be employed (see Karl T Ulrich, 2003) this is not surprising - that a range exists implies predisposition of methods to specific circumstances and learning. Across methods there is a stronger contribution to the form (KD5) and programme use (KD1) dimensions, suggesting a primary mode of learning from prototypes aligns with geometry and utility against the design function, as is likely to be desired in an engineering prototyping process. Some dimensions, namely lifecycle (KD10), character (KD8), environment (KD2), and explanation (KD9) received either lower ratings or higher variability in ratings, suggesting a lower capability of prototyping methods to contribute towards them. Should knowledge in these dimensions be required, care in selection of prototyping methods may be preferable. Of particular note is the low score received by lifecycle (KD10), indicating that product considerations such as sustainability may not be well covered by the prototyping methods and therefore need to be considered through other means within the product development process.

Selection and sequencing of prototyping methods: Prototyping typically requires a sequence of physical and virtual iterations at varying fidelities (Goudswaard et al., 2021 - under review). Where prototyping for a specific design requires contribution across dimensions or a specific focus may be required, there may be a set of prototyping methods more suited to generation of this knowledge at the level of fidelity the designer is currently working. For example, at low fidelities hand-processes are rated higher than machine, while virtual 3D modelling processes are as high or higher in all dimensions except programme use (KD1). Drawing methods are rated higher in informative rather than representative dimensions, highlighting a challenge in representation of geometry and function. At high fidelities, there is a switch to higher ratings for machine-based processes over hand-based in physical, while virtual 3D modelling maintains a high rating. Given such potential per-method differences, a given scenario may be best suited to a specific or subset of methods depending on the knowledge dimensions required. Further, prototyping methods are not equal in cost, time commitment, or skill requirement (Mathias et al., 2019). The balancing of knowledge produced by methods against such variables may also allow improved sequencing and selection of methods, whereby a range of variables may be balanced against the required dimensions to meet process goals (i.e. reduced cost and time).

Study Limitations: While this study has produced statistically significant results, it used a comparatively small sample size and should be extended with further participants. In particular, extending the range of industries and backgrounds of raters would provide interesting views on the variance associated with each knowledge dimension and any relationships between variation and coder expertise. Findings as presented hence provide direction, but require further exploration. Further, the findings of the study should be validated against real prototyping processes as followed by designers in order to validate the patterns that the study implies. These limitations form the basis for an ongoing study.

There is substantial possibility to extend results through further characterisation of the methods against further variables such as process stage, and process performance metrics including cost, quality, and time commitment. Findings here presented are context-neutral, and it is likely that method selection and knowledge dimension rating may vary depending on the cost and time implications of method use.

Future work: Several areas of future work are implied by the findings, in addition to extensions to the study discussed above. First, as methods vary in cost, skill, complexity, and time, it would be highly beneficial to perform a deeper characterisation of the cost/benefit of each across various design prototyping scenarios, such that a guide to better method selection may be produced. In so doing, designers may be guided towards methods and sequences of methods that are i) lowest cost, ii) quickest, iii) provide the most complete, or iv) highest quality or contextually-appropriate contribution. Second, it is the act of prototyping that in many cases supports generation of knowledge, and hence the transition between iterations and methods that may prove of particular value. This implies the importance of sequencing of prototype methods, whereby the transition between different forms and types may create emergent learning. Specific study and characterisation of method sequencing and combination in context of the cumulative addition to knowledge dimensions may support development of better prototyping processes and tools. Finally, examination of the variation in methods across design scenarios would better inform the extent to which knowledge dimensions may be used to classify method output. 


\section{CONCLUSION}

With myriad prototyping techniques available, each with different media, method, and utility, that which a designer selects to perform their prototyping activity potentially holds a large impact over the learnings that are generated. Concurrently, designers often find it challenging to know which techniques to use and when they should be employed. Using the concept of Knowledge Dimensions, this paper presents a study relating prototyping methods to the form of information that they generate, thereby identifying the relationship between methods and knowledge. A rating study was performed, evidencing first that prototyping methods are not equal, with many showing strength in their contribution to geometric and function-based knowledge, but weakness in wider knowledge such as lifecycle. Further results investigated methods across a high/low fidelity and physical/virtual boundary, and suggested equivalence in some physical and virtual methods, and a switch in strength from hand-based to machine-based methods as fidelity increases. Through such findings, this paper begins to form a body of knowledge by which designers may better select methods with respect to the knowledge they require, and by which researchers may better understand the relationships between prototyping activities, methods, and knowledge, and support better structuring of the prototyping process.

\section{ACKNOWLEDGMENTS}

The work reported in this paper has been undertaken as part of the Twinning of digital-physical models during prototyping project. The work was conducted at the University of Bristol, Design and Manufacturing Futures Laboratory (http://www.dmf-lab.co.uk) which is funded by the Engineering and Physical Sciences Research Council (EPSRC), Grant reference (EP/R032696/1).

\section{REFERENCES}

Bogers, Marcel and Willem Horst (July 2014). "Collaborative Prototyping: Cross-Fertilization of Knowledge in Prototype-Driven Problem Solving”. In: Journal of Product Innovation Management 31.4, pp. 744-764. DOI: 10.1111/jpim.12121.

Buchenau, Marion and Jane Fulton Suri (2000). "Experience prototyping”. In: Proceedings of the 3rd conference on Designing interactive systems: processes, practices, methods, and techniques, pp. 424-433.

Camburn, Bradley et al. (2017). "Design prototyping methods: State of the art in strategies, techniques, and guidelines”. In: Design Science 3.Schrage 1993, pp. 1-33. DOI: 10.1017/dsj.2017.10.

Camere, S. and M. Bordegoni (2016). "A lens on future products: An expanded notion of prototyping practice". In: Proceedings of International Design Conference, DESIGN DS 84.21100509737, pp. 155-164.

Engstrom, Stephen P (2009). The form of practical knowledge: A study of the categorical imperative. Harvard University Press.

Gero, John S. (Dec. 1990). “Design Prototypes: A Knowledge Representation Schema for Design”. In: $A I$ Magazine 11.4, pp. 26-26. DOI: 10.1609/AIMAG.V11I4.854.

Goudswaard, Mark et al. (2021 - under review). "Characterising the prototyping practices of Design companies in the South-West of the UK". In: ICED 2021 conference proceedings.

Houde, Stephanie and Charles Hill (1997). “Chapter 16 - What do Prototypes Prototype?” In: Handbook of Human-Computer Interaction (Second Edition). Ed. by Marting G. Helander, Thomas K. Landauer, and Prasad V. Prabhu. Second Edition. Amsterdam: North-Holland, pp. 367-381. DOI: https://doi. org/10.1016/B978-044481862-1.50082-0.

Lim, Youn Kyung, Erik Stolterman, and Josh Tenenberg (2008). "The anatomy of prototypes: Prototypes as filters, prototypes as manifestations of design ideas". In: ACM Transactions on Computer-Human Interaction 15.2. DOI: 10.1145/1375761.1375762.

Mathias, David et al. (2019). "Accelerating product prototyping through hybrid methods: Coupling 3D printing and LEGO”. In: Design Studies 62, pp. 68-99. DOI: 10.1016/j.destud.2019.04.003.

Schon, Donald A and Glenn Wiggins (1992). "Kinds of seeing and their functions in designing”. In: pp. 135-156. Ullman, David G (1992). The mechanical design process. Vol. 2. McGraw-Hill New York.

Ulrich, Karl T (2003). Product design and development. Tata McGraw-Hill Education.

Wall, Matthew B., Karl T. Ulrich, and Woodie C. Flowers (1992). "Evaluating prototyping technologies for product design”. In: Research in Engineering Design 3.3, pp. 163-177. DOI: 10.1007/BF01580518.

Wittgenstein, Ludwig (2009). Philosophical investigations. John Wiley \& Sons. 